Brief Report

\title{
Fear of Falling in Patients With Hip Fractures: Prevalence and Related Psychological Factors
}

\author{
Jan Visschedijk MD, MPH ${ }^{\mathrm{a}, \mathrm{b}, *}$, Romke van Balen MD, PhD ${ }^{\mathrm{a}}$, Cees Hertogh MD, $\mathrm{PhD}^{\mathrm{c}}$, \\ Wilco Achterberg MD, $\mathrm{PhD}^{\mathrm{a}}$ \\ a Department of Public Health and Primary Care, Leiden University Medical Center, Leiden, The Netherlands \\ ${ }^{\mathrm{b}}$ Zorggroep Solis, Deventer, The Netherlands \\ ${ }^{\mathrm{c}}$ Department of Nursing Home Medicine Amsterdam, Free University Medical Center/Institute for Research in Extramural Medicine, Amsterdam, The Netherlands
}

Keywords:

Hip fractures

rehabilitation

fear of falling

falls efficacy

\begin{abstract}
A B S T R A C T
Objective: To determine the prevalence of fear of falling (FoF) in patients after a hip fracture, to investigate the relation with time after fracture, and to assess associations between FoF and other psychological factors. Design: Cross-sectional study performed between September 2010 and March 2011 in elderly patients after a hip fracture.

Setting: Ten postacute geriatric rehabilitation wards in Dutch nursing homes.

Participants: A total of 100 patients aged $\geq 65$ years with a hip fracture admitted to a geriatric rehabilitation ward.

Measurements: FoF and related concepts such as falls-related self-efficacy, depression, and anxiety were measured by means of self-assessment instruments.

Results: Of all patients, 36\% had a little FoF and $27 \%$ had quite a bit or very much FoF. Scores on the Falls Efficacy Scale-International were 30.6 in the first 4 weeks after hip fracture, 35.6 in the second 4 weeks, and 29.4 in the period $\geq 8$ weeks after fracture. In these 3 periods, the prevalence rates of FoF were $62 \%$, $68 \%$, and 59\%, respectively. Significant correlations were found between FoF and anxiety $(P<.001)$, and self-efficacy $(P<.001)$.

Conclusion: In these patients with a hip fracture, FoF is common and is correlated with anxiety and falls-related self-efficacy. During rehabilitation, FoF is greatest in the second 4 weeks after hip fracture. More studies are needed to explore the determinants of FoF and develop interventions to reduce FoF and improve outcome after rehabilitation.
\end{abstract}

Published by Elsevier Inc. on behalf of the American Medical Directors Association, Inc.
The burden of hip fractures on the individual and society is considerable and will continue to increase in the future. ${ }^{1,2}$ Psychological factors are important for successful rehabilitation after hip fracture. ${ }^{3}$ Fear of falling (FoF) is such a factor, which may have more impact on functional recovery than pain or depression. ${ }^{4}$ A recent literature review revealed that our knowledge of FoF in older people recovering from a hip fracture is limited. ${ }^{5}$ Most studies suffer from selection bias because frail older people with substantial comorbidity are frequently excluded. Therefore, we conducted a study in patients with hip fracture in 10 postacute geriatric rehabilitation wards of nursing homes in the Netherlands.

In the Netherlands, almost half of the patients with a hip fracture (mostly frail elderly) recover in postacute geriatric rehabilitation

\footnotetext{
The authors declare no conflicts of interest.

* Address correspondence to Jan Visschedijk, MD, MPH, Department of Public Health and Primary Care, Leiden University Medical Centre, PO Box 9600, 2300 RC Leiden, The Netherlands.

E-mail address: jan.visschedijk@zorggroepsolis.nl (J. Visschedijk).
}

wards of nursing homes. The rehabilitation protocols for these wards are similar, in terms of procedures and inputs. After admission, a multidisciplinary rehabilitation care plan is made by the elderly care physician. This physician is specially trained in medical care of frail elderly and is part of the staff of the nursing home. ${ }^{6}$ Patients generally follow a 4- to 16-week rehabilitation program, which includes wound care, treatment of pain and comorbidity, training in activities of daily living, and occupational and physical therapy. When required, a social worker, psychologist, or dietician is consulted. Patients are discharged when they can function independently or with assistance of formal or informal care at home. Most patients continue some form of physical therapy after discharge. Patients with little comorbidity or complications who only need physical therapy after a hip fracture are usually discharged home after hospital admission. Patients who already live in a nursing home are usually not admitted to a postacute geriatric rehabilitation ward.

The aim of this cross-sectional study was to determine the prevalence of FoF using different instruments, to investigate the relation 
Table 1

Fear of Falling and Falls-Related Self-Efficacy in Different Periods of Rehabilitation

\begin{tabular}{|c|c|c|c|c|}
\hline Characteristics & $\begin{array}{l}\text { First four weeks ( } \leq 28 \text { days) } \\
\text { after fracture }\end{array}$ & $\begin{array}{l}\text { Second four weeks ( } 28-56 \text { days) } \\
\text { after fracture }\end{array}$ & $\begin{array}{l}\text { More than } 8 \text { weeks ( } \geq 57 \text { days) } \\
\text { after fracture) }\end{array}$ & $P$ \\
\hline No. in group & 26 & 40 & 34 & \\
\hline Days after fracture, range & $7-28$ & $29-56$ & $57-292$ & \\
\hline Mean days after fracture (median) & $21.0(22)$ & $42.2(42)$ & $87.7(73.5)$ & \\
\hline Mean FES-I score $(\mathrm{CI})^{*}$ [range] & $30.6(27.0-34.2)[16-46]$ & $35.6(32.2-39.0)[19-60]$ & $29.4(26.7-32.1)[17-52]$ & .025 (Kruskal-Wallis test) \\
\hline FoF one-item, \% with $\mathrm{FoF}^{\dagger}$ & $62 \%$ & $68 \%$ & $59 \%$ & .731 (Pearson $\chi^{2}$ test) \\
\hline Mean GSE (CI) [range] & $22.9(20.5-25.4)[8-30]$ & $21.0(19.2-22.9)[8-30]$ & $21.0(18.7-23.3)[5-30]$ & \\
\hline Mean VAS (CI) [range] & $2.5(1.7-3.3)[0-6]$ & $3.1(2.4-3.8)[0-8]$ & $2.3(1.6-3.1)[0-7]$ & \\
\hline Mean GDS (CI), [range] & $0.2,(0.0-0.3),[0-1]$ & $0.7,(0.2-1.2),[0-7]$ & $1.4,(0.6-2,1),[0-7]$ & \\
\hline Mean HADS-A (CI), [range] & $2.4,(1.1-3.7),[0-13]$ & $2.7,(1.5-3.8),[0-18]$ & $3.5,(2.1-4.9),[0-14]$ & \\
\hline
\end{tabular}

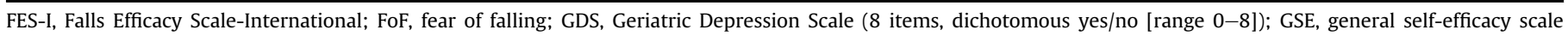

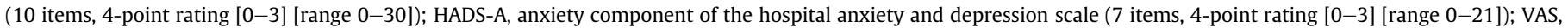
visual analog scale-pain (11-point numerical rating [range 0-10]).

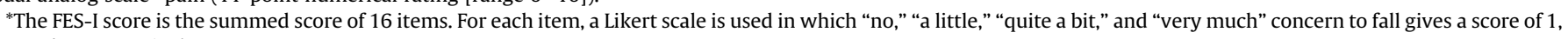
2,3 , and 4 , respectively.

†Patients with FoF answered to the question "Are you afraid of falling?" with "a little," "quite a bit," or "very much."

with time after fracture, and to assess associations between FoF and other psychological factors.

\section{Methods}

A total of 124 patients were eligible to participate. Inclusion criteria were age $\geq 65$ years and admitted to the ward because of a hip fracture. Twenty-four patients were excluded because they were unable to adequately respond to the questions, did not give consent to participate, or had communication problems. Data were collected cross sectionally at every site during a period of 2 weeks. In the first week, the investigators provided information to the participants and health workers. In the second week, the interviews with the participants and tests by physiotherapists were conducted. Additional data were collected via questionnaires issued to the physicians and responsible nurses. Every patient rehabilitating after hip fracture on that rehabilitation ward was eligible to participate.

The Medical Ethical Commission of the VU University Medical Center approved the study and the protocol. All participants provided written informed consent.

Because different types of measures, that is, multi-item self-efficacy and single-item FoF measures, are available for FoF, two instruments were used in the study: the Falls Efficacy Scale-International (FES-I) and the one-item FoF instrument. ${ }^{7}$ The FES-I reflects concern about falling when performing 16 activities of daily living tasks. ${ }^{8}$ The response to the FES-I consists of four levels ranging from "no concern" to "very much concern." 9 The one-item FoF instrument asks one question: "Are you afraid of falling?" and has four answer options: "not at all," "a little," "quite a bit," and "very much."7

To assess FoF in relation to the rehabilitation phase, we divided the participants into 3 groups depending on the number of days between fracture and assessment: phase $1=\leq 28$ days, phase $2=$ 29-56 days, and phase $3=\geq 57$ days. These cutoff points ensured comparable numbers of participants in each group and are also clinically relevant for the rehabilitation process. In phase 1 , the focus is on strength and balance training, in phase 2, on walking independently, and in phase 3 , on increasing walking distance and adjusting to circumstances at home.

Depressive symptoms were measured using the Geriatric Depression Scale 8-item version. ${ }^{10}$ Anxiety was assessed using the anxiety component of the hospital anxiety and depression scale (HADS-A). ${ }^{11}$ Self-efficacy was measured using the Dutch translation of the general self-efficacy scale. ${ }^{12}$ This 10 -item scale measures the general competence of a person to cope with a broad scope of challenging encounters. Pain was assessed by asking the patients to indicate their level of pain on a visual analog scale ranging from 0 to
10. ${ }^{13}$ Analyses were performed using SPSS for Windows, version 17 (SPPS Inc, Chicago, Illinois).

\section{Results}

Of the 100 participants, mean age was 83.1 years and $75 \%$ were female. The mean FES-I score was 32.2. The scores for the FoF 1-item were: no FoF, 37.0\%; a little FoF, 36.0\%; quite a bit FoF, 23.0\%; and very much FoF, 4.0\%. The Pearson correlation between the FES-I and the 1 -item FoF instrument was $0.668(P<.001)$.

Table 1 shows that the percentage of patients with FoF (measured with the FES-I and the FoF 1-item instrument) is greatest in phase 2 of the rehabilitation process. In phases 1,2 , and 3, the FES-I is 30.6, 35.6, and 29.4, respectively ( $P=.025$, Kruskal-Wallis test).

The Pearson correlation between the Geriatric Depression Scale 8-item version and the FES and the 1 -item FoF instrument was $0.111(P=$ $.271)$ and $0.190(P=.058)$, respectively. The Pearson correlation between the general self-efficacy scale and the FES and the 1-item FoF instrument was $-0.295(P=.003)$ and $-0.363(P<.001)$, respectively. The Pearson correlation between the anxiety component of the HADS and the FES, and the 1-item FoF instrument was $0.267(P=.007)$ and $0.359(P<.001)$, respectively. The Pearson correlation between visual analog scale-pain and the FES and the 1-item FoF instrument was 0.250 $(P=.012)$ and $0.152(P=.131)$, respectively.

\section{Discussion}

This study shows that FoF is common among patients after a hip fracture. When using a simple 1 -item instrument to assess FoF, $63 \%$ of the patients had at least some FoF. This is within the broad range of $21 \%$ to $85 \%$ reported in other studies, mainly focusing on communitydwelling older persons. ${ }^{14}$

The mean FES-I of 32.2 in our group is similar to that in a German study of geriatric rehabilitation inpatients in which FES-I was 32 on admission to hospital and 34 at 4 -month follow-up. ${ }^{15}$ In a Dutch study (among mostly independently living older people), the mean score was 26.7 for those aged 70 to 79 years, and 33.0 for those aged $\geq 80$ years. ${ }^{16}$ This indicates that also when using the FES-I as a proxy for FoF, FoF is a considerable clinical problem in rehabilitation after hip fracture.

When measuring in different phases of rehabilitation, FoF and FES-I were highest in the group that had rehabilitated 4 to 8 weeks. Studies are required in which individual participants are followed longitudinally to confirm these results and draw further conclusions.

FoF was strongly associated with anxiety and self-efficacy; however, it is not clear how this relationship is established. Anxiety might be 
a general characteristic of a person and, as such, may facilitate FoF in general. Similarly, a person's lack of self-efficacy about not falling may enhance FoF. The exact features of this relationship, and how they might be modified, needs to be examined in future studies.

A limitation of this study is that the data are cross-sectional, meaning that the individual patients were not followed throughout the rehabilitation process. This implies that the different subgroups may not be fully comparable. Patients who rehabilitated at a faster rate may have been discharged earlier and were probably underrepresented; this may have resulted in overestimation of the prevalence of FoF. Although the patients included in this study constitute a large proportion of the (often frail) older people who recover after a hip fracture, caution is required when generalizing the results to other groups.

\section{Conclusion}

FoF is common among patients with a hip fracture, using different measurement instruments, and is related to other psychological factors, such as anxiety and depression. The prevalence was greatest in the group rehabilitating between 28 and 56 days. However, the exact prevalence during different phases in the rehabilitation process has to be further explored in longitudinal studies. This information is necessary to develop interventions to diminish FoF to improve functional capacity and participation after hip fractures.

\section{References}

1. Zidén L, Wenestam CG, Hansson-Scherman M. A life-breaking event: Early experiences of the consequences of a hip fracture for elderly people. Clin Rehabil 2008;22:801-811.

2. Burge R, Dawson-Hughes B, Solomon DH, et al. Incidence and economic burden of osteoporosis-related fractures in the United States, 2005-2025. J Bone Miner Res 2007;22:465-475.
3. Mossey JM, Mutran E, Knott K, Craik R. Determinants of recovery 12 months after hip fracture: The importance of psychological factors. Am J Public Health 1989;79:279-286.

4. Oude Voshaar RC, Banerjee S, Horan M, et al. Fear of falling more important than pain and depression for functional recovery after surgery for hip fracture in older people. Psychol Med 2006;36:1635-1645.

5. Visschedijk J, Achterberg W, Van Balen R, Hertogh C. Fear of falling after hip fracture: A systematic review of measurement instruments, prevalence, interventions, and related factors. J Am Geriatr Soc 2010;58:1739-1748.

6. Schols JM, Crebolder HF, van Weel C. Nursing home and nursing home physician. The Dutch experience. J Am Med Dir Assoc 2004;5:207-212.

7. Jorstad E, Hauer K, Becker C, et al. Measuring the psychological outcomes of falling: A systematic review. J Am Geriatr Soc 2005;53:501-510.

8. Yardley L, Beyer N, Hauer K, et al. Development and initial validation of the Falls Efficacy Scale-International (FES-I). Age Ageing 2005;34:614-619.

9. Kempen GI, Todd CJ, Van Haastregt JC, et al. Cross-cultural validation of the Falls Efficacy Scale International (FES-I) in older people: Results from Germany, the Netherlands and the UK were satisfactory. Disabil Rehabil 2007;29: 155-162.

10. Gerritsen DL, Jongenelis K, Pot AM, et al. The GDS-8; a short, client- and userfriendly shortened version of the Geriatric Depression Scale for nursing homes. Tijdschr Gerontol Geriatr 2007;38:298-304.

11. Spinhoven P, Ormel J, Sloekers PP, et al. A validation study of the Hospital Anxiety and Depression Scale (HADS) in different groups of Dutch subjects. Psychol Med 1997;27:363-370.

12. Teeuw B, Schwarzer R, Jerusalem M. Dutch adaptation of the general self-efficacy scale. 1994. Available at: http://userpage.fu-berlin.de/ health/ dutch.htm. Last accessed October 2, 2012.

13. Ohnhaus EE, Adler R. Methodological problems in the measurement of pain: A comparison between the verbal rating scale and the visual analogue scale. Pain 1975;1:379-384.

14. Scheffer AC, Schuurmans MJ, Dijk van N, et al. Fear of falling: Measurement strategy, prevalence, risk factors and consequences among older persons. Age Ageing 2008;37:19-24.

15. Denkinger MD, Igl W, Lukas A, et al. Relationship between fear of falling and outcomes of an inpatient geriatric rehabilitation population-fear of the fear of falling. J Am Geriatr Soc 2010;58:664-673.

16. Kempen GI, Todd CJ, Van Haastregt JC, et al. Cross-cultural validation of the Falls Efficacy Scale International (FES-I) in older people: Results from Germany, the Netherlands and the UK were satisfactory. Disabil Rehabil 2007;29: 155-162. 